\title{
Corrigendum: Two-step cell polarization in algal zygotes
}

Kenny A. Bogaert, Tom Beeckman and Olivier De Clerck

Nature Plants 3, 16221 (2017); published 23 January 2017; corrected 13 February 2017.

In the version of this Letter originally published, the first affiliation for Tom Beeckman should have read: VIB-UGent Center for Plant Systems Biology, Technologiepark 927, B-9052 Ghent, Belgium. This has been corrected in all versions of the Letter. 